Submitted to the Monthly Notices of the Royal Astronomical Society

Preprint typeset using $\mathrm{IAT}_{\mathrm{E} X} \mathrm{X}$ style emulateapj v. 12/16/11

\title{
OPTICAL EMISSION ASSOCIATED WITH THE GALACTIC SUPERNOVA REMNANT G179.0+2.6
}

\author{
Thomas G. How ${ }^{1}$, Robert A. Fesen ${ }^{2}$, Jack M. M. Neustadt ${ }^{2}$, Christine S. Black $^{2}$, And Nicolas Outters $^{3}$ \\ submitted to the Monthly Notices of the Royal Astronomical Society
}

\begin{abstract}
Narrow passband optical images of the large Galactic supernova remnant G179.0+2.6 reveal a faint but nearly complete emission shell dominated by strong [O III] 4959,5007 $\AA$ line emission. The remnant's optical emission, which consists of both diffuse and filamentary features, is brightest along its southern and northeastern limbs. Deep $\mathrm{H} \alpha$ images detect little coincidence emission indicating an unusually high [O III] $/ \mathrm{H} \alpha$ emission ratio for such a large and apparently old remnant. Low-dispersion optical spectra of several regions confirm large $[\mathrm{O}$ III] $/ \mathrm{H} \alpha$ line ratios with typical values around 10 . The dominance of [O III] emission for the majority of the remnant's optical filaments suggests shock velocities above $100 \mathrm{~km} \mathrm{~s}^{-1}$ are present throughout most of the remnant, likely reflecting a relatively low density ambient ISM. The remnant's unusually strong [O III] emission adds to the remnant's interesting set of properties which include a thick radio emission shell, radial polarization of its radio emission like that typically seen in young supernova remnants, and an unusually slow-rotating gammaray pulsar with a characteristic spin-down age $\simeq 50 \mathrm{kyr}$.
\end{abstract}

Subject headings: ISM: individual objects: G179.0+2.6, ISM: supernova remnant - shock waves optical

\section{INTRODUCTION}

Of the nearly 300 currently confirmed Galactic supernova remnants (SNRs) (Green 2014), the majority were initially identified in the radio due to nonthermal radio emissions associated with shocked gas (Milne 1970. Downes 1971). Of these, only $\sim 30 \%$ exhibit coincident optical emission.

Here we report the discovery of extensive optical emission associated with the large (diameter $\sim 70^{\prime}$ ) shell-type Galactic SNR G179.0+2.6. This remnant was first detected in the radio at 1.42 and $2.695 \mathrm{GHz}$ by Fürst \& Reich (1986) who noted a triple polarized source near its center, which was later determined to be a background radio galaxy with bipolar jets (Fürst et al. 1989).

The remnant's radio emission consists of an unusually thick shell (see Fig. 1) compared to similarly large and old SNRs. It has an estimated diameter $\simeq 70$ pc assuming a distance estimate of $\sim 3.5 \mathrm{kpc}$ based on its surface brightness and the $\Sigma-D$ relation of Milne (1979). Such a size implies an old remnant with an age $>10^{4}$ yr (Fürst et al. 1989). A radio source near its center is a gamma-ray pulsar (PSR J0554+3107) with an unusually long spin period of $0.465 \mathrm{~s}$ with a characteristic age of $\simeq 52 \mathrm{kyr}$ (Pletsch et al. 2013).

While the remnant's overall radio emission spectral in$\operatorname{dex} \alpha=-0.45 \pm 0.11$ (Gao et al. 2011) is unremarkable for an old SNR, its radial projected magnetic field is unexpected and more typical of young SNRs (Fürst \& Reich 1986 Fürst et al. 1989). Gao et al. (2011), confirmed the radial arrangement of the remnant's magnetic field and suggested the Galactic magnetic field in the remnant's area might be oriented along the line of sight as a possible explanation, although they noted this was unlikely.

\footnotetext{
${ }^{1}$ Curdridge Observatory, Southhampton UK

2 Department of Physics \& Astronomy, Dartmouth College, Hanover, NH 03755 USA

3 Orange Observatory, Haute-Savoie France
}

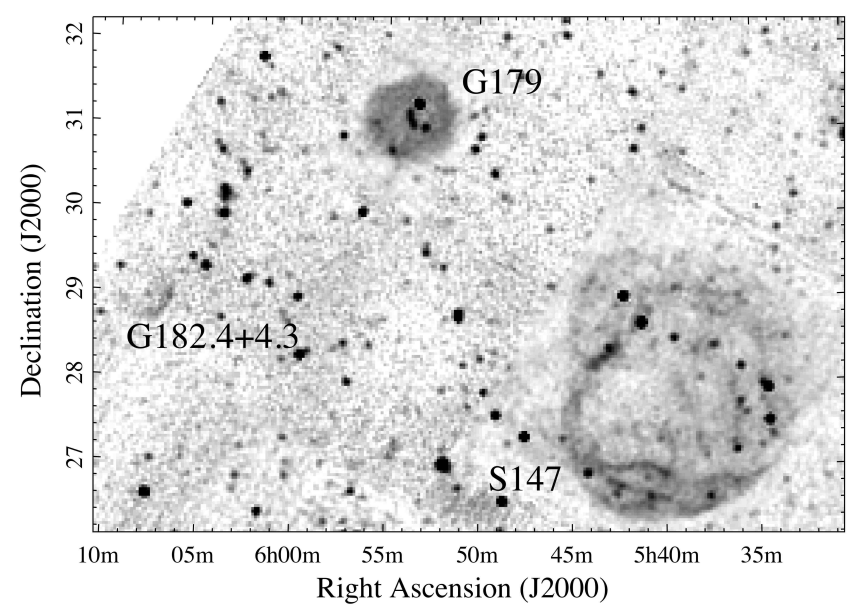

FIG. 1. $-2695 \mathrm{MHz}$ radio image of G179.0+2.6 and its surroundings from Fürst et al. (1990), highlighting its unusually thick radio emission shell relative to the nearby SNRs S147 and G182.4+4.3.

There has been no previously reported optical emission associated with the G179.0+2.6 SNR (hereafter referred to as simply G179). No coincident emission is visible on the Palomar Sky Surveys (DSS1 and DSS2), or the emission-line survey of the Galactic plane of Parker et al. (1979). However, a partial shell of faint $\mathrm{H} \alpha$ emission can be seen at the remnant's position in the Virginia Tech Spectral Line Survey (VTSS) of the Galactic Plane (Dennison et al. 1998; Finkbeiner 2003) and small patches of emission filaments especially along the remnant's northeastern limb are visible in the Isaac Newton Telescope (INT) Photometric H $\alpha$ Survey (IPHAS) of the Northern Galactic Plane (Drew et al. 2005, González-Solares et al. 2008).

One of us (TH) obtained deep $\mathrm{H} \alpha$ and [O III] filter images of G179 in a search of associated optical emission. These images revealed a surprisingly complete [O III] emission shell coincident with the remnant's radio shell. Despite similarly deep $\mathrm{H} \alpha$ images, little coincident $\mathrm{H} \alpha$ 

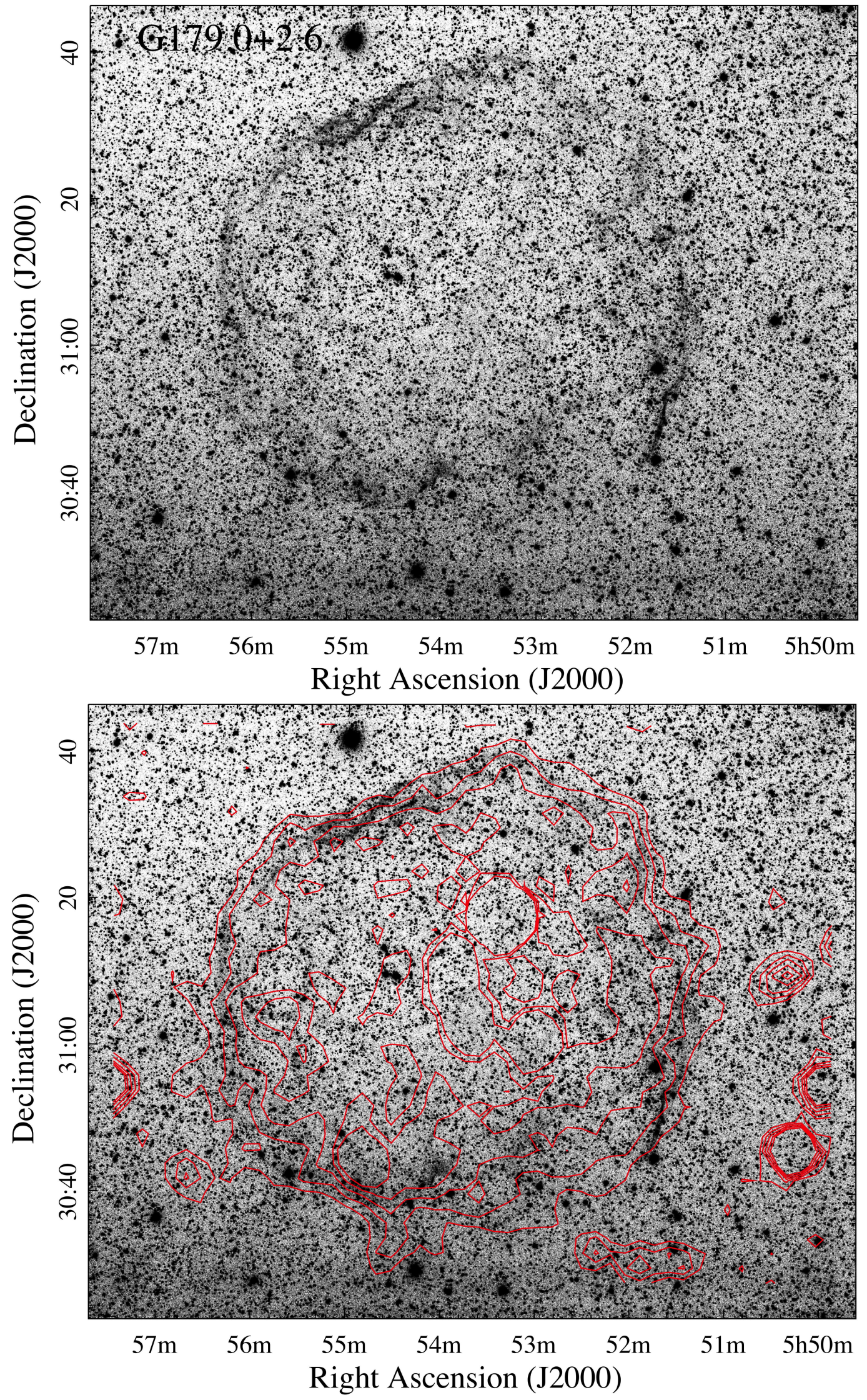

Fig. 2.- Top: A 9.2 hour Curdridge Observatory [O III] $5007 \AA$ image of G179.0+2.6 showing a nearly complete shell of emission. Bottom: Same image with overlay of the $100 \mathrm{~m}$ Effelsberg $2695 \mathrm{MHz}$ brightness temperature contours (Fürst et al. 1990). 
emission was detected.

This discovery was followed-up by additional $[\mathrm{O}$ III $]$ and $\mathrm{H} \alpha$ images plus low-dispersion optical spectra of a few representative filaments. Optical images and spectra of G179 are described in $\S 2$ with results presented in $\S 3$ and discussed in $\S 4$.

\section{OBSERVATIONS}

Discovery of wide-spread optical emission from the G179 supernova remnant was made in November 2016 using a $90 \mathrm{~mm}$ refractor at the Curdridge Observatory in Southampton, UK. Images were taken using a Astrodon $30 \AA$ FWHM filter and a Atik 490 camera employing a Sony ICX814 CCD which was binned $2 \times 2$ yielding $1690 \times 1352$ pixels. This system provided a FOV of $64^{\prime} \times 86^{\prime}$ with an image scale of $3 . ! 72$ pixel $^{-1}$.

Combining 22 dithered 1500 s exposures for a total exposure time of $9.2 \mathrm{hr}$, faint [O III] emission was detected coincident with the remnant's radio emission. Similarly long $30 \AA$ FWHM H $\alpha$ filter exposures showed little coincident emission.

Follow-up images were obtained using a $0.4 \mathrm{~m}$ telescope at Orange Observatory in eastern France. Narrow passband $\mathrm{H} \alpha$ and [O III] images (FWHM = $30 \AA$ ) were taken using a Astrodon and a Moravian G4 camera with a KAF-16803 CCD with $4096 \times 4096$ pixels. This system yielded a FOV of $83^{\prime} \times 83^{\prime}$ with an image scale of $1^{\prime \prime} .22$ pixel $^{-1}$.

A series of 35 dithered $600 \mathrm{~s} \mathrm{H} \alpha$ images and 66 dithered $600 \mathrm{~s}$ [O III] images were obtained between December 2016 and March 2017. These were then combined into a $5.8 \mathrm{hr} \mathrm{H} \alpha$ exposure and a $11 \mathrm{hr}$ [O III] exposure of the remnant.

Guided by these wide-field images of the remnant's [O III $]$ emission, additional images of the remnant's northeastern and southwestern limbs were obtained in Feburary and March 2017 using the 1.3m McGraw-Hill telescope at MDM Observatory at Kitt Peak, AZ. These were taken using a [O III] filter $(\mathrm{FWHM}=50 \AA)$ using either a $2 \mathrm{k} \times 2 \mathrm{k}$ or a $1 \mathrm{k} \times 1 \mathrm{k}$ SITe CCDs. On-chip binning yielded an image scale of $1.06 \operatorname{arcsec}_{\text {pixel }}{ }^{-1}$. The image of the SW region was obtained from $4 \times 1500$ s exposures, while two overlapping NE regions were obtained using $2 \times 1200$ s exposures and taken under thin cloud conditions.

Low-dispersion spectra of four filamentary regions in the NE and SW were obtained in January and February 2017 with the $2.4 \mathrm{~m}$ Hiltner telescope at MDM Observatory and the OSMOS Spectrograph (Martini et al. 2011). Using a blue VPH grism $(\mathrm{R}=1600)$, single $2000 \mathrm{~s}$ exposures were taken of four filamentary regions covering 3900-6800 ^ with a spectral resolution of $1.2 \AA$ pixel $^{-1}$.

Standard pipeline data reduction of MDM images and spectra using IRAF was preformed. Direct images were bias-subtracted, flat-field corrected using twilight sky flats, and averaged to remove cosmic rays and improve signal-to-noise. Spectra were similarly reduced using IRAF and the software L.A. Cosmic (van Dokkum 2001) to remove cosmic rays. Spectra were calibrated using an Ar lamp and spectroscopic standard stars (Oke 1974; Massey \& Gronwald 1990).

\subsection{Images}

Figure 2 shows G179's [O III] emission structure along with an overlay of the remnant's radio emission contours as seen by the Effelsberg $100 \mathrm{~m}$ radio telescope at $11 \mathrm{~cm}$ $(2695 \mathrm{MHz})$ (Fürst et al. 1990). The remnant's optical emission forms a nearly complete shell, brightest along its northeastern, southern, and southwestern limbs. Although most of the [O III] emission is diffuse, some sharp filaments are present along the northeast and southwest limb regions as shown in Figure 3.

Figure 4 shows a comparison of the [O III] and $\mathrm{H} \alpha$ images of G179. These images make it clear that the remnant's optical emission is dominated by [O III] emission. Except for the northeastern limb, little $\mathrm{H} \alpha$ emission is detected along most of the remnant's radio shell. This helps explain why the remnant was missed in previous $\mathrm{H} \alpha$ surveys of Galactic SNRs searching for associated optical emission. Not visible in the [O III] images, some exceedingly faint and diffuse $\mathrm{H} \alpha$ emission appears present inside the remnant's lower weatern interior region.

In the few regions where some $\mathrm{H} \alpha$ emission is detected, its morphology is different from that of the remnant's [O III] emission. For example, as shown in the lower panels of Figure 4 for the remnant's northeastern limb area, one finds little correspondence between filaments bright in [O III] to those bright in $\mathrm{H} \alpha$. The broad and extended "V" shaped $\mathrm{H} \alpha$ emission feature seen here from the IPHAS image survey (lower right panel) exhibits little coincident [O III] emission. Conversely, the bright [O III] filament some $30^{\prime \prime}$ to the north from this $\mathrm{H} \alpha$ filament is largely absent in $\mathrm{H} \alpha$ emission. Weak $\mathrm{H} \alpha$ line emission is a property present throughout the remnant's extensive [O III] emission structure.

\subsection{Optical Spectra}

Due to the remnant's faint optical emissions, lowdispersion spectra were only obtained of the remnant's brighter northeastern and southwestern filaments. Slit positions are indicated in Figure 3, with the resulting spectra presented in Figure 5. Observed emission line fluxes relative to $\mathrm{H} \alpha$ are listed in Table 1.

The $[\mathrm{O}$ III $]$ and $\mathrm{H} \alpha$ images of G179 had already suggested unusually high $[\mathrm{O} \mathrm{III}] / \mathrm{H} \alpha$ ratios and this was confirmed by spectra. We measured values around 10 for three of the four regions sampled (see Table 1). The lack of detected $\mathrm{H} \beta 4861 \AA$ emission prevents tabulating the strength of line emissions relative to $\mathrm{H} \beta$, the usual ratio used to express the relative strength of [O III] emission in SNRs.

In places where the optical emission was bright enough to detect $[\mathrm{S}$ II $]$ line emission, we find $[\mathrm{S}$ II $] / \mathrm{H} \alpha$ ratios $>0.4$, i.e., above the standard criteria for identifying shock emission (D'Odorico et al. 1980, Blair et al. 1981 Dopita et al. 1984; Fesen et al. 1985). However, the remnant's faintness prevented a firm measurement of the ratio of the density sensitive [S II] $6716,6731 \AA$ emission lines. For one slit position, namely NE 1a, we measured a $6716 / 6731$ ratio $\simeq 1.30$ which indicates a density close to the low density limit, $\leq 100 \mathrm{~cm}^{-3}$ (Osterbrock \& Ferland 2006). 

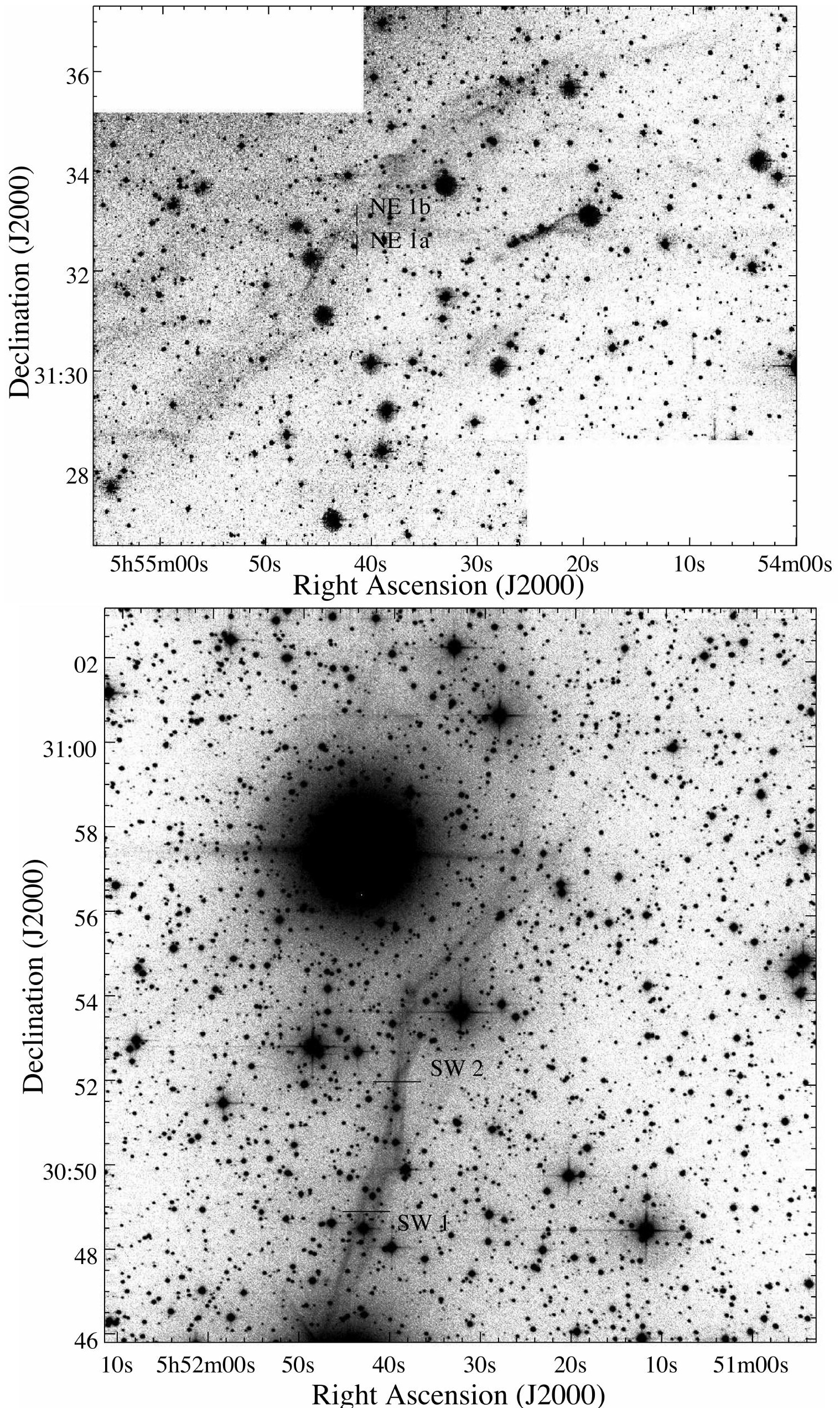


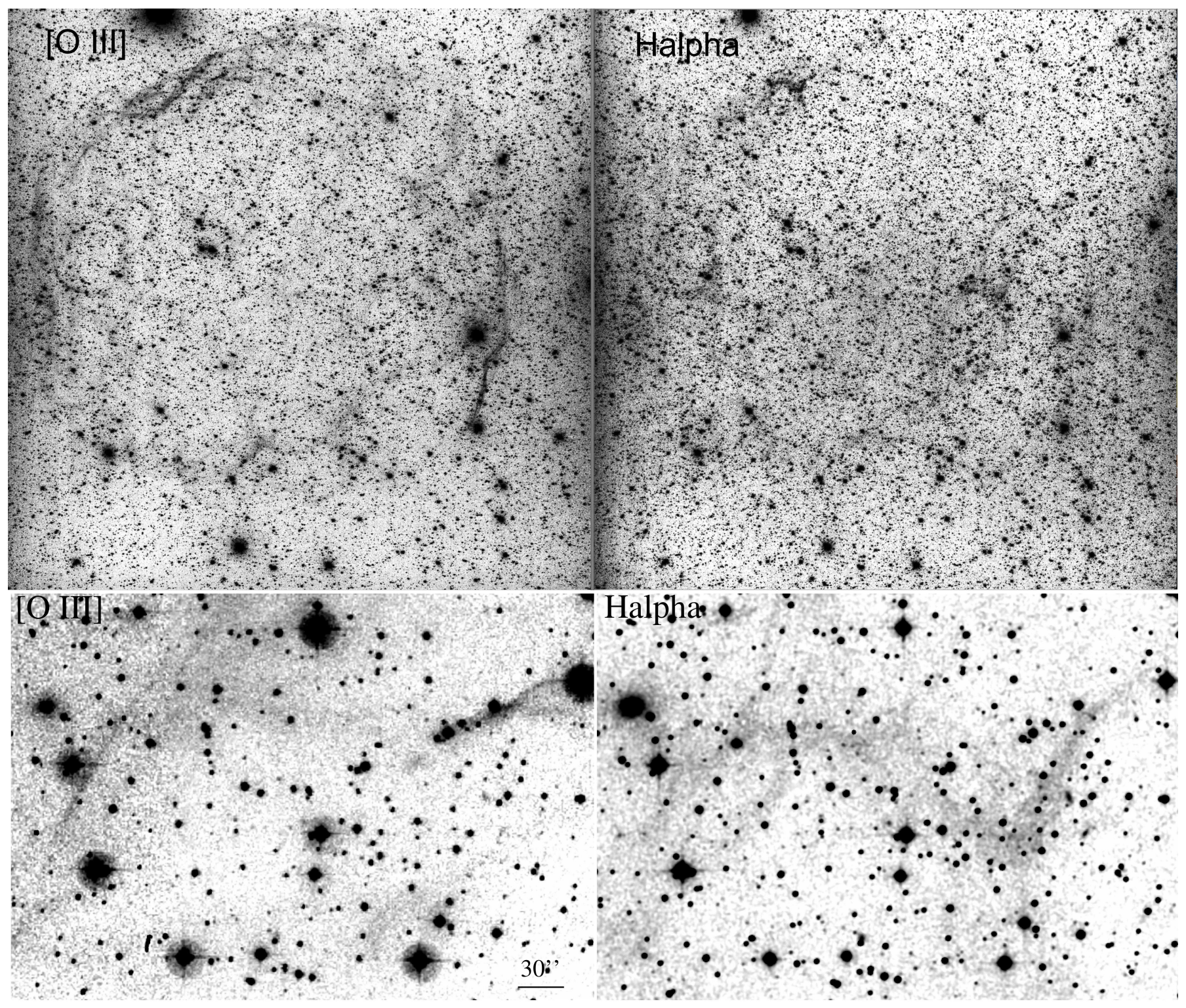

Fig. 4.- Comparison of G179.0+2.6 emissions in [O III] and H $\alpha$. Top Panels: A 11 hour long exposure of the remnant in [O III] (left) and a 5.8 hour $\mathrm{H} \alpha$ image (right) as detected in the $0.4 \mathrm{~m}$ Orange Observatory telescope. North is up, and East is to the left. The majority of the remnant's optical emission is dominated by strong [O III] emission with only a small northeastern filamentary region bright in $\mathrm{H} \alpha$. (Note: Extreme east and west central edges of both images are contaminated by light leaks.) Bottom Panels: Comparison of the remnant's northeastern limb region as seen in a MDM 1.3m [O III] image and in the IPHAS H $\alpha$ image.

With an angular diameter of $\simeq 70^{\prime}$, G179 ranks among the largest Galactic SNRs known. Moreover, at a distance of $\sim 3.5 \mathrm{kpc}($ Fürst \& Reich 1986), this translates into a linear diameter $\approx 70 \mathrm{pc}$, indicative of a relatively old SNR and one likely in the later Sedov-Taylor phase of adiabatic expansion. The presence of an unusually slowly rotating gamma-ray pulsar, PSR J0554+3107, with a characteristic spin down age around $52 \mathrm{kyr}$ (Pletsch et al. 2013) is consistent with the picture of an well evolved SNR.

On the other hand, optical emission that is so dominated by [O III] line emission is relatively rare in Galactic remnants, especially large and old ones like G179. This is because $[\mathrm{O} \mathrm{III}] / \mathrm{H} \alpha$ ratios around 2 or larger are generated by relatively fast shocks above $\approx 100 \mathrm{~km} \mathrm{~s}^{-1}$ (Raymond 1979; Shull \& McKee 1979, Hartigan et al.
1987), unusual in large and highly evolved SNRs. Moreover, for there to be weak associated $\mathrm{H} \alpha$ emission, the shock's recombination zone must be 'incomplete', meaning the entire postshock cooling zone is not fully established. Such incomplete shocks are often identified by $[\mathrm{O} \mathrm{III}] / \mathrm{H} \beta$ ratios greater than 10 and commonly associated with shocks in excess of $100 \mathrm{~km} \mathrm{~s}^{-1}$.

In the case of the optical emission seen in G179 where the emission is so faint, $\mathrm{H} \beta$ emission was not readily detected at any of our four slit positions, meaning [O III] $4959+5007 / \mathrm{H} \beta$ ratios are likely quite high, exceeding 20 for positions SW 2 and NW 1b. Such strong [O III] emission filaments have been seen in filaments in a few remnants (Hester et al. 1994). One example lies in the Cygnus Loop where a filament along the remnant's far eastern boundary shows a $[\mathrm{O} \mathrm{III}] / \mathrm{H} \beta$ of 45 , near the up- 


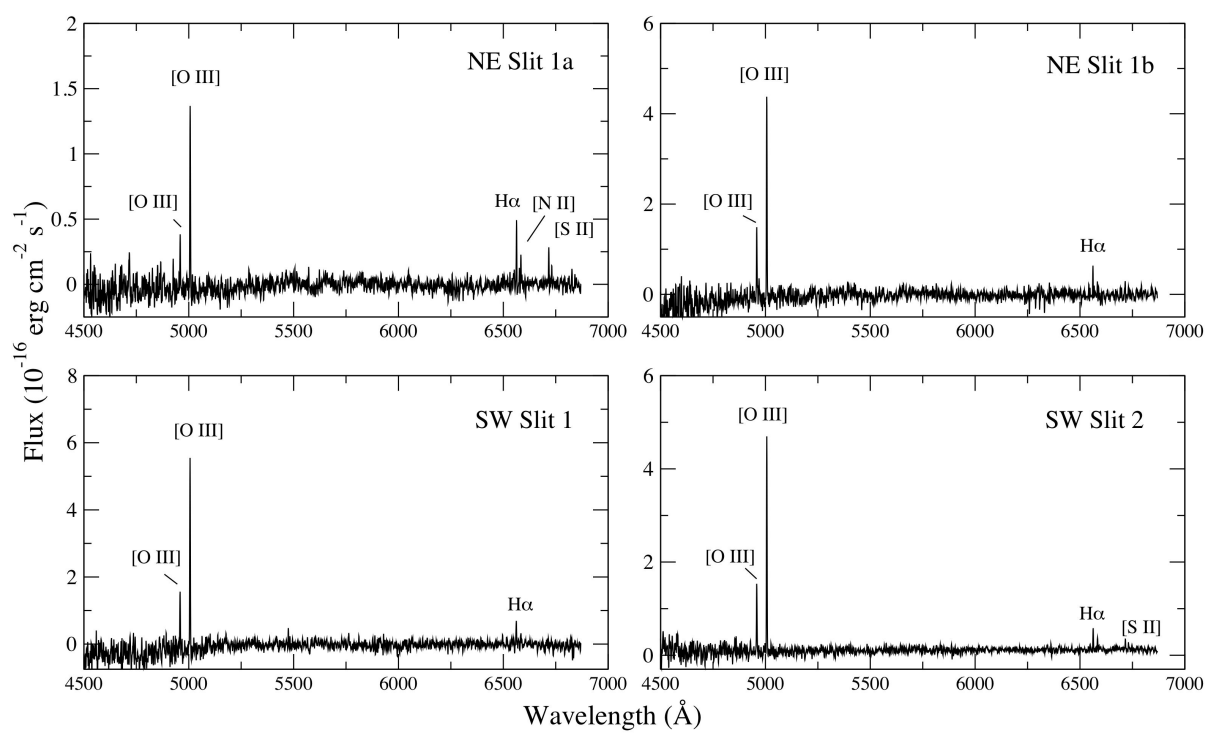

FIG. 5. - Spectra of four regions in G179.0+2.6. See Figure 3 for locations of slit positions.

TABLE 1

Relative FluXes in G179.0+2.6

\begin{tabular}{|c|c|c|c|c|}
\hline Line/Ratio & SW 1 & SW 2 & NW 1a & NW 1b \\
\hline $\mathrm{H} \beta 4861$ & $<100$ & $<50$ & $<20$ & $<50$ \\
\hline [O III] 4949 & 280 & 380 & 110 & 330 \\
\hline [O III 5007 & 910 & 1150 & 390 & 1010 \\
\hline Н $\alpha 6563$ & 100 & 100 & 100 & 100 \\
\hline [N II] 6583 & 40 & 52 & 40 & 33 \\
\hline$[$ S II] 6716 & $<25$ & 44 & 56 & $<30$ \\
\hline [S II] 6731 & $<25$ & 34 & 25 & $<30$ \\
\hline$[\mathrm{S} \quad \mathrm{II}] / \mathrm{H} \alpha$ & (2) & 0.78 & $(0.80)$ & $\ldots$ \\
\hline [S II] $6716 / 6731$ & $\ldots$ & 1.30 & $>1.3$ & $\ldots$ \\
\hline$\rho\left(\mathrm{cm}^{-3}\right)$ & $\ldots$ & $<100$ & $<100$ & $\ldots$ \\
\hline H $\alpha$ flux ${ }^{a}$ & 2.1 & 1.4 & 1.3 & 1.5 \\
\hline
\end{tabular}

${ }^{a}$ Flux units: $10^{-16} \mathrm{erg} \mathrm{s}^{-1} \mathrm{~cm}^{-2}$.

per end of the range observed in SNRs (Fesen \& Hurford 1996). It is believed this filaments represents material only recently shocked by the remnant's $300+\mathrm{km} \mathrm{s}^{-1}$ shock front and has not yet had time to establish a full recombination zone.

However, examples of SNRs whose whole optical emission is dominated by [O III] line emission are rare. One large and old galactic SNR whose optical emission is like that of $\mathrm{G} 179$, is $\mathrm{G} 65.3+5.7$. It exhibits strong [O III] emission in almost all of its optical structure. This remnant was only discovered through deep [O III] images (Gull et al. 1977), and subsequently confirmed by radio observations (Reich et al. 1979). At an estimaged distance of $0.8-1.0 \mathrm{kpc}$ (Gull et al. 1977; Reich et al. 1979; Rosado 1981, Sitnik et al. 1983), its $3.3 \times 4$ degree angular size translates to a physical size $\sim 70 \mathrm{pc}$, similar to G179.

Except for a small filamentary region known as Sharpless 91 (S91; Sharpless 1959) along the southern limb of its $4 \times 3.3$ degree shell, most of G65.3+5.7's filaments show large $[\mathrm{O} \mathrm{III}] / \mathrm{H} \beta$ ratios consistent with incomplete recombination zones and shock velocities above $90 \mathrm{~km}$ $\mathrm{s}^{-1}$ (Fesen et al. 1985, Mavromatakis et al. 2002). The one noticeable exception is the $\mathrm{H} \alpha$ bright filament, $\mathrm{S} 91$. This situation is similar to what is seen in G179 whose only bright $\mathrm{H} \alpha$ emission is a few filaments along the remnant's northeastern limb (see Fig. 4).

Based on [O III] line profiles, Boumis et al. (2004) estimated a global expansion velocity for $\mathrm{G} 65.3+5.7$ of $\approx 155$ $\mathrm{km} \mathrm{s}^{-1}$. This value is consistent with a relatively highshock velocity through its structure despite its old age. A similar analysis and shock velocity probably applies to G179.

For both G65.3+5.7 and G179, a relatively low surrounding ambient density is likely the cause for their optical emission to be nearly dominated by strong [O III] filaments, an otherwise rare property in older SNRs. A low density surrounding ISM, as has been suggested in the case of G65.2+5.7 caused by a pre-SN progenitor wind-blown cavity (Xiao et al. 2009), would also help explain why such [O III] emission dominate remnants are also so optically faint.

The distance to G179 is uncertain. A value of $3.5 \mathrm{kpc}$ was cited by Fürst \& Reich (1986) based on the $\Sigma-D$ relation given by Milne (1979). However, $\Sigma-D$ derived distances are controversial and widely viewed as not being especially accurate (Green 1991, Dubner \& Giacani 2015). Indeed, a search of recent $\Sigma-D$ distance estimates for G179 finds a wide range of estimated distance values; Case \& Bhattacharya (1998) lists $6.1 \mathrm{kpc}$, Guseinov et al. (2003) cite a value of $2.9 \mathrm{kpc}$, while Pavlovic et al. (2014) lists a distance of $3.1 \mathrm{kpc}$ but with a large uncertainty range of $2.1-8.2 \mathrm{kpc}$.

Although its true distance is currently uncertain, G179 is unlikely to lie at distances much greater than $\sim 5 \mathrm{kpc}$. The remnant's [O III] emission is so strong relative to $\mathrm{H} \alpha$, foreground extinction toward the remnant is likely to be modest, a conclusion consistent with its anti-center galactic location. Reddening at $l=179.0 \pm 0.3, b=2.6 \pm$ 0.3 based on a three-dimensional map presented in Green et al. (2015), which utilizes Pan-STARRS (Schlafly et al. 2014) and 2MASS (Skrutskie et al. 2006 photometry, indicate G179 lies in a region of relatively low extinction with $E(B-V)$ values around 0.2 at $1 \mathrm{kpc}$ rising above 0.4 around $5 \mathrm{kpc}$. Values of $E(B-V)$ much greater than 0.3 would raise the $[\mathrm{O} \mathrm{III}] / \mathrm{H} \alpha$ above that seen in even extreme cases of incomplete recombination. 
In addition, at a distance of $5 \mathrm{kpc}$ the remnant's diameter would exceed $100 \mathrm{pc}$ making it one the physically largest known Galactic SNRs. Such a size would be hard to understand given the requirement of a shock velocity well in excess of $100 \mathrm{~km} \mathrm{~s}^{-1}$ enabling it to exhibit incomplete recombination in the postshock cooling zone.

\section{CONCLUSIONS}

We have discovered substantial optical emission from the large Galactic supernova remnant G179.0+2.6 which previously had no known associated optical emission. Narrow passband filter images reveal a nearly complete but faint emission shell dominated by strong [O III] line emission. Equally deep $\mathrm{H} \alpha$ images detect little coincidence emission indicating an unusually widespread high $[\mathrm{O}$ III $] / \mathrm{H} \alpha$ ratio for such a large and apparently old remnant. Low-dispersion optical spectra of several re- gions confirm large $[\mathrm{O} \mathrm{III}] / \mathrm{H} \alpha$ line ratios, suggesting incomplete cooling in the postshock recombination zone. The remnant's unusually strong [O III] emission suggests shock velocities above $100 \mathrm{~km} \mathrm{~s}^{-1}$ throughout most of the remnant, likely reflecting a relatively low density ambient ISM.

We thank Sakib Rasool for helpful communications regarding optical emission associated with G179, and the MDM Observatory staff for their excellent instrument assistance. This research was made possible by funds from the NASA Space Grant, the Jonathan Weed Fund, the Denis G. Sullivan Fund, and Dartmouth's School of Graduate and Advance Studies.

\section{REFERENCES}

Blair, W. P., Kirshner, R. P., \& Chevalier, R. A. 1981, ApJ, 247, 879

Boumis, P., Meaburn, J., López, J. A., et al. 2004, A\&A, 424, 583

Case, G. L., \& Bhattacharya, D. 1998, ApJ, 504, 761

Dennison, B., Simonetti, J. H., \& Topasna, G. A. 1998, Publications of the Astronomical Society of Australia, 15, 147

Dopita, M. A., Binette, L., Dodorico, S., \& Benvenuti, P. 1984, ApJ, 276, 653

D’Odorico, S., Dopita, M. A., \& Benvenuti, P. 1980, A\&AS, 40, 67 Downes, D. 1971, AJ, 76, 305

Drew, J. E., Greimel, R., Irwin, M. J., et al. 2005, MNRAS, 362, 753

Dubner, G., \& Giacani, E. 2015, A\&A Rev., 23, 3

Fesen, R. A., Blair, W. P., \& Kirshner, R. P. 1985, ApJ, 292, 29

Fesen, R. A., \& Hurford, A. P. 1996, ApJS, 106, 563

Finkbeiner, D. P. 2003, ApJS, 146, 407

Fürst, E., \& Reich, W. 1986, A\&A, 154, 303

Fürst, E., Reich, W., Kuehr, H., \& Stickel, M. 1989, A\&A, 223, 66

Fürst, E., Reich, W., Reich, P., \& Reif, K. 1990, A\&AS, 85, 691

Gao, X. Y., Han, J. L., Reich, W., et al. 2011, A\&A, 529, A159

González-Solares, E. A., Walton, N. A., Greimel, R., et al. 2008, MNRAS, 388, 89

Green, D. A. 1991, PASP, 103, 209

Green, D. A. 2014, Bulletin of the Astronomical Society of India, 42,47

Green, G. M., Schlafly, E. F., Finkbeiner, D. P., et al. 2015, ApJ, 810,25

Gull, T. R., Kirshner, R. P., \& Parker, R. A. R. 1977, ApJ, 215, L69

Guseinov, O. H., Ankay, A., Sezer, A., \& Tagieva, S. O. 2003, Astronomical and Astrophysical Transactions, 22, 273

Hartigan, P., Raymond, J., \& Hartmann, L. 1987, ApJ, 316, 323

Hester, J. J., Raymond, J. C., \& Blair, W. P. 1994, ApJ, 420, 721

Lorimer, D. R., Lyne, A. G., \& Camilo, F. 1998, A\&A, 331, 1002

Martini, P., Stoll, R., Derwent, M. A., et al. 2011, PASP, 123, 187

Massey, P., \& Gronwald, C. 1990, ApJ, 358, 344
Mavromatakis, F., Boumis, P., Papamastorakis, J., \& Ventura, J. 2002, A\&A, 388, 355

Milne, D. K. 1970, Australian Journal of Physics, 23, 425

Milne, D. K. 1979, Australian Journal of Physics, 32, 83

Oke, J. B. 1974, ApJS, 27, 21

Osterbrock, D. E., \& Ferland, G. J. 2006, Astrophysics of gaseous nebulae and active galactic nuclei, 2nd. ed. Sausalito, CA: University Science Books

Parker, R. A. R., Gull, T. R., \& Kirshner, R. P. 1979, NASA SP-434

Pavlovic, M. Z., Dobardzic, A., Vukotic, B., \& Urosevic, D. 2014, Serbian Astronomical Journal, 189, 25

Pletsch, H. J., Guillemot, L., Allen, B., et al. 2013, ApJ, 779, L11

Raymond, J. C. 1979, ApJS, 39, 1

Renaud, M., Marandon, V., Gotthelf, E. V., et al. 2010, ApJ, 716, 663

Reich, W., Berkhuijsen, E. M., \& Sofue, Y. 1979, A\&A, 72, 270

Reich, W. 2002, in Proceedings of the 270. WE-Heraeus Seminar on Neutron Stars, Pulsars, and Supernova Remnants, MPE Report 278 eds. W. Becker, H. Lesch, \& J. Trümper, pg 1

Rosado, M. 1981, ApJ, 250, 222

Sabbadin, F., \& D'Odorico, S. 1976, A\&A, 49, 119

Schlafly, E. F., Green, G., Finkbeiner, D. P., et al. 2014, ApJ, 789,15

Sharpless, S. 1959, ApJS, 4, 257

Shull, J. M., \& McKee, C. F. 1979, ApJ, 227, 131

Skrutskie, M. F., Cutri, R. M., Stiening, R., et al. 2006, AJ, 131, 1163

Stupar, M., Parker, Q. A., \& Filipović, M. D. 2008, MNRAS, 390, 1037

Sitnik, T. G., Klementeva, A. Y., \& Toropova, M. S. 1983,

Soviet Ast., 27, 292

van Dokkum, P. G. 2001, PASP, 113, 1420

Xiao, L., Reich, W., Fürst, E., \& Han, J. L. 2009, A\&A, 503, 827 